\title{
INTOXICAÇÃO POR CHUMBO: UTILIZAÇÃO DE MÍDIAS SOCIAIS COMO FERRAMENTA DE EDUCAÇÃO EM SAÚDE DO TRABALHADOR
}

\author{
LEAD POISONING: USE OF SOCIAL MEDIA AS AN EDUCATION \\ TOOL FOR OCCUPATIONAL HEALTH
}

\author{
Paola Frassinetti de Oliveira Correia ${ }^{1}$ \\ Gabriela Portela ${ }^{2}$ \\ Júlia Maria Vasconcelos Dias Moura ${ }^{3}$ \\ Marília Juliane Pedrosa Gurgel ${ }^{4}$ \\ Maurizene Rodrigues Silva ${ }^{5}$ \\ Nathalia de Oliveira Burgo ${ }^{6}$
}

RESUMO: O chumbo é um metal extremamente tóxico, quando em altas doses, para o ser humano. A contaminação ocorre principalmente pela falta de uso de equipamentos de proteção individual no manejo dos materiais contendo chumbo. Pensando na problemática que atinge os trabalhadores de indústrias e outros profissionais, foi criado em 2005 a Política Nacional de Saúde do Trabalhador, passando a ser principal organizadora da saúde do trabalhador no SUS. Como meios que podem ser utilizados para colocar em prática estratégias de educação em saúde, o digital ganha cada vez mais espaço. Diante disso, esse estudo objetiva relatar a construção e alcance de materiais digitais para mídias sociais de educação em saúde do trabalhador acerca da intoxicação por chumbo. Foi realizado um estudo de desenvolvimento de tecnologia educativa na Faculdade de Enfermagem Nossa Senhora das Graças da Universidade de Pernambuco. Os recursos de transmissão de informação elaborados para divulgação nas mídias

\footnotetext{
1 Discente do 9o módulo do curso de Enfermagem da Faculdade de Enfermagem Nossa Senhora das Graças da Universidade de Pernambuco (UPE).

2 Discente do 9o módulo do curso de Enfermagem da Faculdade de Enfermagem Nossa Senhora das Graças da Universidade de Pernambuco (UPE).

${ }^{3}$ Discente do 9o módulo do curso de Enfermagem da Faculdade de Enfermagem Nossa Senhora das Graças da Universidade de Pernambuco (UPE).

${ }^{4}$ Discente do 9o módulo do curso de Enfermagem da Faculdade de Enfermagem Nossa Senhora das Graças da Universidade de Pernambuco (UPE).

${ }^{5}$ Discente do 9o módulo do curso de Enfermagem da Faculdade de Enfermagem Nossa Senhora das Graças da Universidade de Pernambuco (UPE).

${ }^{6}$ Discente do 9o módulo do curso de Enfermagem da Faculdade de Enfermagem Nossa Senhora das Graças da Universidade de Pernambuco (UPE).
} 
sociais foram lançados no Instagram $\AA$ e no Facebook $\AA$, sendo alcançados 1788 curtidas, 1145 visualizações, 188 compartilhamentos e 327 comentários. Com isso, a campanha se tornou aliada ao fortalecimento do pensar crítico e reflexivo da comunidade virtual. Também permitiu evidenciar a necessidade da abordagem do tema em diversos âmbitos, objetivando a sensibilização da população sobre o tema e a promoção e educação em saúde.

Palavras chave: Enfermagem. Intoxicação por Chumbo. Mídias Sociais. Saúde do Trabalhador.

ABSTRACT: Lead is an extremely toxic metal, when in high doses, for humans. Contamination occurs mainly due to the lack of use of personal protective equipment in the handling of materials containing lead. Thinking about the problem that affects workers in industries and other professionals, in 2005 the National Policy for Occupational Health was created, becoming the main organizer of occupational health in SUS. As a means that can be used to put health education strategies into practice, digital is gaining more and more space. In view of this, this study aims to report the construction and reach of digital materials for social media on health education of workers about lead poisoning. A study on the development of educational technology was carried out at the Faculdade de Enfermagem Nossa Senhora das Graças at the University of Pernambuco. The information transmission resources designed for dissemination on social media were launched on Instagram ${ }^{\circledR}$ and Facebook ${ }^{\circledR}$, reaching 1788 likes, 1145 views, 188 shares and 327 comments. With that, the campaign became associated with the strengthening of critical and reflective thinking of the virtual community. It also made it possible to highlight the need to approach the theme in several areas, aiming to raise the population's awareness of the theme and health promotion and education.

Keywords: Nursing. Lead Poisoning. Social Media. Occupational health. 


\section{INTRODUÇÃO}

Saturnismo, Plumbismo ou Intoxicação por chumbo é um tipo de contaminação ocupacional e ambiental, já sendo descrito a mais de 2000 anos. O chumbo é um metal pesado bastante flexível, com baixo ponto de fusão, e ao mesmo tempo extremamente tóxico, quando em altas doses, para o ser humano (BRASIL, 2006). Ele pode ser encontrado nos mais diversos materiais, por exemplo, em canos utilizados em construções, baterias automobilísticas e também como um dos componentes de corantes das tinturas para cabelo, batons, tintas de parede, entre outros (ROCHA et al, 2017).

Os efeitos desse tipo de contaminação são considerados extensos e de difícil diagnóstico, pois o metal afeta praticamente todos os órgãos e sistemas do corpo, apresentando sintomatologia como náuseas, vômitos, mal-estar, dores abdominais e cansaços, levando a doenças como anemia, transtornos mentais, problemas na tireoide, hipertensão e problemas renais. Como pode-se notar, a sintomatologia torna-se bastante inespecífica, podendo ser facilmente confundida com outras patologias, sendo assim, uma anamnese eficaz é de grande importância para detectar a intoxicação (DUARTE et al, 2016; MARTINS, 2016).

Um dos públicos mais prejudicados no Brasil por esse tipo de intoxicação são os profissionais das indústrias que utilizam desse composto químico e os trabalhadores da área de construção e reformas. A contaminação, que pode acontecer pela inalação, ingestão ou quando em contato frequente com a pele, ocorre principalmente pela falta de uso de equipamentos de proteção individual no manejo dos materiais contendo chumbo (TERÇARIOL, 2017; SOUZA et al, 2016).

Pensando na problemática que atinge esses trabalhadores e outros profissionais que podem acabar por sofrer algum agravo ligado a riscos ocupacionais, foi criado em setembro de 2002 a Rede Nacional de Atenção 
Integral à Saúde do Trabalhador (Renast), por meio da Portaria no 1.679/GM, com objetivo de disseminar ações de saúde do trabalhador, articuladas às demais redes do Sistema Único de Saúde, SUS, sendo redefinida em 2005 como Política Nacional de Saúde do Trabalhador, passando a ser principal organizadora da saúde do trabalhador no SUS. Utilizando-se de estratégias de educação em saúde dentre outras atividades para sensibilizar os trabalhadores, além de direcionar as proteções adequadas que cada profissional deve aderir (BRASIL, 2006).

Como meios que podem ser utilizados para colocar em prática essas estratégias de educação em saúde, o digital vem para ganhar cada vez mais espaço. A comunicação em saúde em meio digital tem por finalidade unir os três direitos básicos do cidadão: informação, saúde e comunicação (BIZ; GUARESCHI, 2017). Com essa premissa, a utilização dos novos meios de comunicabilidade pelo ser humano enfatiza a importância do uso de instrumentos eficazes na difusão de informações, sendo este um espaço de facilidade para novas formas de mobilização social, pois não existe barreiras físicas que impedem o compartilhamento de notícias, atingindo um grande número de público, dos mais variados, e rapidamente (CRUZ et al, 2013).

A internet tornou-se um meio muito utilizado pelos profissionais de saúde para veicular informações acerca de campanhas de saúde, prevenção e conscientização a partir de ferramentas do meio digital. Esse novo patamar de fazer educação em saúde passou a ser intitulado como e-saúde, uma aliada pedagógica ao ensinar e levar assuntos diversos para população, que os compartilha e troca experiências em meio digital (CRUZ et al, 2013; PESSONI; AKERMAN, 2015).

A concepção do conhecimento ocorre a partir da interação entre diferentes agentes sociais, pelo diálogo proporcionado pelas mídias digitais e pela reflexão acerca do que é discutido (CRUZ et al, 2013). Diante disso, esse estudo propõe a criação de mídias que abordem a temática da intoxicação por chumbo ocupacional, como instrumento de comunicação em saúde. 


\section{OBJETIVO}

Relatar a construção e alcance de materiais digitais para mídias sociais de educação em saúde do trabalhador acerca da intoxicação por chumbo.

\section{MÉTODO}

Estudo do tipo desenvolvimento de tecnologia educativa para promoção de uma temática relacionada à saúde do trabalhador, descrevendo ferramentas e técnicas utilizadas durante o processo (MOREIRA, 2013). A construção foi realizada entre o final do mês de agosto e início de setembro, na Faculdade de Enfermagem Nossa Senhora das Graças - Universidade de Pernambuco, como requerimento para nota da unidade temática Informática em Saúde do oitavo módulo.

Para organização do trabalho, foi dividido em algumas etapas. Na primeira etapa, foi sorteado as diferentes temáticas existentes entre os grupos para início da elaboração, o grupo deste trabalho, em questão, ficou com a temática intoxicação por chumbo. Com o conhecimento da temática, foi realizado um estudo das literaturas existentes para maior entendimento do assunto, e decisão do público ao qual seria direcionado os materiais. Ainda nesse primeiro momento, foi possível observar que os trabalhadores de indústrias pesadas e baterias automobilísticas seriam os mais afetados, sendo a eles direcionado.

$\mathrm{Na}$ segunda etapa ocorreu a construção propriamente dita dos materiais. De início foi organizado um roteiro direcionado, tanto para o vídeo, quanto para o podcast (arquivo de multimídia como um programa de rádio, trazendo temáticas das mais diversas), e o infográfico (representação visual gráfica de assuntos complexos), para que todos os três se conversassem e não fugissem dos tópicos que todos deveriam abordar. Nesse momento criou-se uma 
personalidade ao grupo, como as mídias sociais trazem em canais para a caracterização da marca, assim o "canal" passou a ser intitulado de Quinteto Fantástico da Saúde, a arte foi montada através do CorelDRAW $X 7 \AA$.

Já com o roteiro em mãos, organizado pelo estudo realizado na primeira etapa, iniciou-se a montagem do vídeo, do podcast e do infográfico. No infográfico, foi utilizado o Canva® e suas ferramentas. No podcast foram utilizados o MasterRecord® para a gravação, e para a edição e cortes o Audio Joiner®. Já no vídeo utilizou-se da técnica Motion Design, com a movimentação de textos e imagens, tanto em 2D como em 3D, para uma representação mais lúdica, para isso foram utilizados o PowToon® no desenvolvimento do vídeo, o Youtube ${ }^{B}$ para upload do vídeo, o MasterRecord® para gravação das falas, o Clipchamp® ${ }^{\circledR}$ na questão de toda a parte de mixagem de som, e o InShot® para as legendas.

E na terceira etapa, foram realizadas as postagens, entre o dia 01 e 20 de setembro, nas redes sociais dos membros do grupo. Por questão organizacional, o grupo agregou todos os links através do Linktree $\mathbb{B}$. As redes sociais utilizadas foram o Whatsapp®, Facebook $\AA$, Instagram $\AA$, além da utilização do SounCloud® para upload do podcast.

\section{RESULTADOS}

Os recursos de transmissão de informação (vídeo, infográfico e podcast) elaborados para divulgação nas mídias sociais foram lançados no Instagram®e no Facebook® das cinco integrantes do grupo que estão nomeadas no quadro 1 de alcance das mídias sociais como Pérola, Esmeralda, Jade, Cristal e Opala. As publicações foram realizadas no dia 01 de setembro de 2019, desde essa data até o dia 20 de setembro de 2019 foram alcançados 1788 curtidas 1145 visualizações do vídeo 188 compartilhamentos e 327 comentários (Quadro 02).

A divulgação das postagens por whatsapp foi bastante útil para gerar mais envolvimento, levando em consideração que a ausência de impulsionamento faz 
com que o conteúdo não chegue facilmente ao público, que vê a publicação rapidamente entre as atualizações de seu feed de notícias. A rede social de impacto mais perceptível foi o Instagram, algo esperado, por ser uma rede de alto engajamento no mundo atualmente. No que diz respeito ao recurso com maior repercussão o vídeo obteve maior destaque, talvez um dos motivos seja que o infográfico e o podcast são instrumentos mais novos com menor difusão na sociedade.

Dentre os comentários realizados nas postagens, nove foram questionamentos sobre a temática abordada, que variavam desde perguntas mais simples a mais complexas de serem respondidas. Segue abaixo algumas das indagações respondidas pelas integrantes nas redes sociais:

"Quão comum é esse tipo de intoxicação? Quando se compara por exemplo com organofosforados e organoclorados?"

Questionamento na postagem do Vídeo no Facebook® de Pérola.

"Onde posso buscar mais informações sobre a contaminação por chumbo?"

Questionamento na postagem do Infográfico e Podcast no Instagram® de Cristal.

\begin{tabular}{|c|c|c|c|c|}
\hline \multicolumn{5}{|c|}{ Quadro 1 - Alcance nas Midias Sociais de cada Integrante do Grupo } \\
\hline \multicolumn{5}{|c|}{ Integrante do Grupo: Pérola } \\
\hline \multicolumn{5}{|c|}{ Publicação: Infográfico e Podcast } \\
\hline Midia Social & Comentários & Curtidas & Compartilhamentos & Visualizaçōes \\
\hline Facebook & 1 & 6 & - & - \\
\hline Instagram & 8 & 31 & - & - \\
\hline \multicolumn{5}{|c|}{ Publicaçăo: Video } \\
\hline Midia Social & Comentários & Curtidas & Compartilhamentos & Visualizaçōes \\
\hline Facebook & 7 & 13 & 4 & - \\
\hline Instagram & 13 & 34 & - & 58 \\
\hline \multicolumn{5}{|c|}{ Integrante do Grupo: Esmeralda } \\
\hline \multicolumn{5}{|c|}{ Publicaçāo: Infográfico e Podcast } \\
\hline Midia Social & Comentários & Curtidas & Compartilhamentos & Visualizaçōes \\
\hline Facebook & - & 7 & - & - \\
\hline Instagram & 61 & 221 & 72 & - \\
\hline \multicolumn{5}{|c|}{ Publicação: Video } \\
\hline Midia Social & Comentários & Curtidas & Compartilhamentos & Visualizaçōes \\
\hline Facebook & 12 & 33 & 3 & - \\
\hline Instagram & 40 & 137 & 10 & 356 \\
\hline
\end{tabular}


Intoxicação por Chumbo: Utilização de Mídias Sociais como Ferramenta de Educação em Saúde do Trabalhador

Integrante do Grupo: Jade

Publicaçăo: Infográfico e Podcast

\begin{tabular}{|l|c|c|c|c|}
\hline Midia Social & Comentários & Curtidas & Compartilhamentos & Visualizaçōes \\
\hline Facebook & 5 & 12 & - & - \\
\hline Instagram & 3 & 67 & - & - \\
\hline \multicolumn{7}{|c|}{ Publicação: Video } \\
\hline
\end{tabular}

\begin{tabular}{|l|c|c|c|c|}
\hline Midia Social & Comentários & Curtidas & Compartilhamentos & Visualizaçōes \\
\hline Facebook & 11 & 18 & 9 & - \\
\hline Instagram & 56 & 160 & - & 409 \\
\hline
\end{tabular}

Integrante do Grupo: Cristal

Publicação: Infografico e Podcast

\begin{tabular}{|c|c|c|c|c|}
\hline Midia Social & Comentários & Curtidas & Compartilhamentos & Visualizaçōes \\
\hline Facebook & 2 & 14 & 1 & - \\
\hline Instagram & 7 & 47 & - & - \\
\hline \multicolumn{5}{|c|}{ Publicação: Video } \\
\hline Midia Social & Comentários & Curtidas & Compartilhamentos & Visualizaçōes \\
\hline Facebook & 4 & 18 & - & - \\
\hline Instagram & 34 & 89 & - & 205 \\
\hline \multicolumn{5}{|c|}{ Integrante do Grupo: Opala } \\
\hline \multicolumn{5}{|c|}{ Publicação: Infográfico e Podcast } \\
\hline Midia Social & Comentários & Curtidas & Compartilhamentos & Visualizaçōes \\
\hline Facebook & 4 & 16 & 3 & - \\
\hline Instagram & 11 & 60 & - & - \\
\hline \multicolumn{5}{|c|}{ Publicação: Video } \\
\hline Midia Social & Comentários & Curtidas & Compartilhamentos & Visualizaçōes \\
\hline Facebook & 35 & 59 & 28 & - \\
\hline Instagram & 13 & 65 & - & 117 \\
\hline
\end{tabular}

\begin{tabular}{|l|c|c|c|c|}
\hline \multicolumn{6}{|c|}{ Quadro 2 - Alcance Total nas Mídias Sociais do Grupo } \\
\hline \multicolumn{7}{|c|}{ Publicação: Infográfico e Podcast } \\
\hline Mídia Social & Comentários & Curtidas & Compartilhamentos & Visualizações \\
\hline Facebook & 12 & 55 & 4 & 0 \\
\hline Total: & 102 & 481 & 76 & 0 \\
\hline \multicolumn{6}{|c|}{ Publicação: Video } \\
\hline Mídia Social & Comentários & Curtidas & Compartilhamentos & Visualizações \\
\hline Facebook & 69 & 141 & 44 & 0 \\
\hline Intagram & 156 & 485 & 10 & 1145 \\
\hline Total: & 225 & 626 & 54 & 1145 \\
\hline Total Geral: & 327 & $\mathbf{1 7 8 8}$ & $\mathbf{1 8 8}$ & $\mathbf{1 1 4 5}$ \\
\hline
\end{tabular}

A aquisição e abrangência das interações sociais nas publicações, mostra que a utilização de redes sociais online têm impacto positivo no acesso a informação sobre saúde do trabalhador.

Revista Interdisciplinar em Saúde, Cajazeiras, 7 (1): 2039-2050, 2020, ISSN: 2358-7490. 
Evidenciando-se, também, como uma importante ferramenta propagadora de informações essenciais sobre a intoxicação por chumbo. No entanto é preciso reconhecer que não foi possível avaliar se o público atingido nas mídias sociais são trabalhadores propensos ao risco de intoxicação ou se possuem alguma vinculação nas suas redes sociais.

\section{DISCUSSÃO}

A internet tornou-se uma importante plataforma para o compartilhamento de ideias, experiências e conhecimentos, sendo reconhecida como tecnologia de informação e comunicação na sociedade. Nesse meio, as redes sociais e afins são algumas das ferramentas que possibilitam a veiculação das informações no meio digital, visto que a acessibilidade e o entrosamento entre os participantes colabora com a produção e reprodução dos serviços (BIZ; GUARESCHI, 2017).

A proposta da campanha educativa virtual surgiu como um inovador instrumento em prol da elucidação de dúvidas e problemas que fazem parte da comunidade cibernética e consequentemente da comunidade como um todo. Neste sentido, a elaboração e a realização de um projeto de educação em saúde na visão das mídias digitais se orienta como uma metodologia ativa, pois proporcionou autonomia e protagonismo aos discentes no processo de aprendizagem, assim como estimulou o cenário das redes sociais a partir da construção coletiva do conhecimento em seus diferentes saberes (SILVA et al, 2015).

Para o desenvolvimento da campanha sobre Intoxicação por Chumbo pelo grupo, foram pensadas não só o prevenir mas também o promover a saúde e o autocuidado, proporcionando empoderamento através da informação. Como consequência, as estratégias estabelecidas na elaboração do vídeo, do podcast e do infográfico buscaram trabalhar a criatividade com o propósito de deixar os três elementos o mais simples didaticamente e o mais interessante possível aos olhos do público-alvo. 
As vivências na construção do grupo permitiram o aprendizado sobre a temática, até então não tão conhecida pelas integrantes. Logo, a busca e a leitura do artigo, manuais e protocolos possibilitou a discussão sobre a contaminação por chumbo na saúde do trabalhador, como também contribuiu com o incentivo de uma percepção para além da realidade acadêmica de jovens em sua bolha social. Pois para atingir as pessoas nas redes como Facebook®, Instagram® e afins, mais que chamar a atenção e ir de encontro aos que fazem parte do convívio das discentes, foi preciso pensar como gerar impacto sobre o tema em leigos, em conhecidos e não-conhecidos.

Percebeu-se o quanto a temática é pouco abordada e o quanto as abordagens até então existentes já são antigas, embora não ultrapassadas. Ao levar esse ponto em consideração, buscou-se seguir pelo princípio da empatia, ao colocar-se no lugar da pessoa que veria e participaria da campanha. Dessa forma, o grupo experienciou desde o início, na escolha de cores, no roteiro para o vídeo e o podcast, na produção textual para o infográfico, as melhores maneiras de usar as ferramentas de composição e edição em prol de um impacto positivo na educação em saúde. Após a produção e divulgação dos materiais nas redes sociais, a intenção de receber um feedback do público foi possível e também completamente alcançada. $O$ impacto gerado com as publicações nos cinco perfis para além dos números contabilizados nas curtidas e compartilhamentos, foi percebido principalmente na qualidade dos comentários e questionamentos feitos.

Entendendo então que a promoção à saúde objetiva transformar efetivamente condições de vida, a campanha se tornou aliada ao fortalecimento do pensar crítico e reflexivo da comunidade virtual. Em tempos de propagação de notícias falaciosas e desmedidas, divulgar e reproduzir saberes científicos junto ao povo é educar em saúde mas também reafirmar o que é saúde e o porquê é preciso continuar lutando em defesa da mesma.

Diante do exposto, o relato da experiência em saúde do trabalhador através das plataformas digitais provocou sensibilização significativa. Aquém a mensuração do alcance do público e a análise do impacto, o grande desafio foi o uso das ferramentas para a temática estabelecida. 


\section{CONCLUSÃO}

A contaminação por chumbo ainda se configura como um problema de saúde pública, afetando principalmente trabalhadores de indústrias que manuseiam o elemento ou entram em contato com materiais que o contenham. Sendo assim, faz-se necessário a elaboração de instrumentos que ajudem na conscientização e orientação dos trabalhadores sobre os riscos que pode trazer à saúde, como preveni-los e, consequentemente, auxiliando na diminuição da contaminação por chumbo. Para isso, visando a rapidez, facilidade e alcance das informações, as mídias digitais são uma ótima ferramenta para propagação desse conhecimento, através de campanhas leves e de linguagem simples que ajudem na disseminação da informação.

Por esse motivo, o presente estudo objetivou a produção de campanha digital sobre a contaminação de operários por chumbo. Foi possível constatar que as publicações realizadas nas redes sociais tiveram uma boa repercussão, já que a possibilidade de interação rápida e fácil que elas trazem consigo, permitiram aos usuários opinarem, tirarem suas dúvidas e deixarem curiosidades sobre o tema, facilitando a percepção do envolvimento e interesse do público pela temática e a necessidade de abordá-la. Muito embora a campanha tenha conseguido atingir muitas pessoas, não é possível saber se o público atingido são pessoas suscetíveis a intoxicação por chumbo ou tem alguma relação com quem está propenso.

O desenvolvimento deste trabalho possibilitou às acadêmicas um maior conhecimento sobre a intoxicação de operários por chumbo e os seus impactos, bem como a reflexão a respeito de como a utilização das tecnologias em saúde podem ter reflexos positivos na vida e na saúde dos trabalhadores. Além disso, também permitiu evidenciar a necessidade da abordagem do tema em diversos âmbitos, objetivando a sensibilização da população sobre o tema e a promoção e educação em saúde. 


\section{REFERÊNCIAS BIBLIOGRÁFICAS}

BIZ, Osvaldo; GUARESCHI, Pedrinho A. Mídia, Educação e Cidadania. Porto Alegre: Evangraf, 2017.

CRUZ, Daniela Imolesi et al. O uso das mídias digitais na educação em saúde. Cadernos da FUCAMP, v. 10, n. 13, p. 130-142, 2013.

BRASIL. Ministério da Saúde. Secretaria de Atenção à Saúde. Departamento de Ações Programáticas Estratégicas. Atenção à saúde dos trabalhadores expostos ao chumbo metálico / Ministério da Saúde, Secretaria de Atenção à Saúde, Departamento de Ações Programáticas Estratégicas. Brasília: Editora do Ministério da Saúde, p.48, 2006.

DUARTE, B. S. et al. Agentes teratogênicos: mercúrio e chumbo. Cadernos Técnicos de Saúde, v. 2, n. 1, jun. 2016.

MARTINS, Daniela Filipa Quintas de Lemos. Efeitos do cádmio e chumbo na saúde humana. 2016. Tese de Doutorado.

MOREIRA, Camila Brasil et al. Construção de um Vídeo Educativo sobre Detecção Precoce do Câncer de Mama. Revista Brasileira de Cancerologia, Rio de Janeiro, v. 59, n. 3, p. 401- 407, set. 2013.

PESSONI, Arquimedes; AKERMAN, Marco. Percepções de docentes e discentes sobre uso educativo de mídias sociais. ABCS Health Sciences, v. 40, n. 3, p. 178-183, dez. 2015.

ROCHA, Rafaela; PEZZINI, Marina Ferri; POETA, Julia. Fontes de contaminação pelo chumbo e seus efeitos tóxicos na saúde ocupacional. Ciência em Movimento, v. 19, n. 39, p. 23-32, maio 2017.

SILVA, Luciana Saraiva et al. Formação de profissionais críticos-reflexivos: o potencial das metodologias ativas de ensino aprendizagem e avaliação na aprendizagem significativa. Revista del Congrés Internacional de Docència Universitària i Innovació (CIDUI), n. 2, 2015.

SOUZA, Valmir; KONRAD, Odorico; JUNIOR, Affonso Celso Gonçalves. Contaminação por chumbo, riscos, limites legais e alternativas de remediação. Veredas do Direito: Direito Ambiental e Desenvolvimento Sustentável, v. 13, n. 25, p. 249-276, 2016.

TERÇARIOL, Simone Galbiati. A intoxicação por chumbo como patologia ocupacional. Fisioterapia Brasil, v. 11, n. 4, p. 309-314, 2017. 